\title{
Zero point survey: a multidisciplinary idea to STEP UP resuscitation effectiveness
}

\author{
Cliff Reid ${ }^{1}$, Peter Brindley ${ }^{2}$, Chris Hicks ${ }^{3}$, Simon Carley ${ }^{4}$, Clare Richmond ${ }^{1}$, \\ Michael Lauria ${ }^{5}$, Scott Weingart ${ }^{6}$ \\ ${ }^{1}$ Greater Sydney Area Helicopter Emergency Medical Service, Bankstown, NSW, Australia \\ ${ }^{2}$ Department of Critical Care Medicine, University of Alberta, Edmonton, AB, Canada \\ ${ }^{3}$ Department of Emergency Medicine, St. Michael's Hospital, Toronto, ON, Canada \\ ${ }^{4}$ Emergency Department, Manchester Royal Infirmary, Manchester Metropolitan University, Manchester, \\ United Kingdom \\ ${ }^{5}$ Geisel School of Medicine, Dartmouth College, Hanover, NH, USA \\ ${ }^{6}$ Department of Emergency Medicine, Stony Brook University Hospital, Stony Brook, NY, USA
}

The primary survey assessment is a cornerstone of resuscitation processes. The name itself implies that it is the first step in resuscitation. In this article, we argue that in an organized resuscitation the primary survey must be preceded by a series of steps to optimize safety and performance and set the stage for the execution of expert team behavior. Even in the most time critical situations, an effective team will optimize the environment, perform self-assessments of personal readiness and participate in a preemptive team brief. We call these processes the 'zero point survey' as it precedes the primary survey. This paper explains the rationale for the zero point survey and describes a structured approach designed to be suitable for all resuscitation situations.

Keywords Resuscitation; Communication

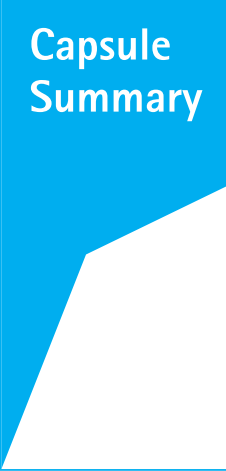

What is already known

Standard resuscitation teaching begins with the primary survey. Although life support courses acknowledge some aspects of non-technical skills, these are rarely taught in a simple, structured, and memorable way, and often leave out environmental considerations.

\section{What is new in the current study}

We propose that prior to performing a primary survey, attention is made to modifiable personal, team and environmental factors to optimize resuscitation. We have named this the zero point survey.
elSSN: 2383-4625

Received: 28 August 2017

Revised: 13 September 2017

Accepted: 13 September 2017

Correspondence to: Cliff Reid Greater Sydney Area Helicopter Emergency Medical Service, NSW Ambulance Rescue Helicopter Base, 33 Nancy Ellis Leebold Drive, Bankstown Airport, NSW 2200, Australia E-mail: reidcg@me.com

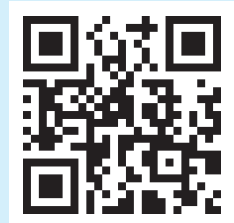

How to cite this article:

Reid C, Brindley P, Hicks C, Carley S, Richmond C, Lauria M, Weingart S. Zero point survey: a multidisciplinary idea to STEP UP resuscitation effectiveness. Clin Exp Emerg Med 2018;5(3):139-143.

This is an Open Access article distributed under the terms of the Creative Commons Attribution Non-Commercial License (http:// creativecommons.org/licenses/by-nc/4.0/). 


\section{INTRODUCTION}

The primary survey (PS) is widely trusted and taught as the first step in all resuscitations. This is because, when properly applied, the PS can identify, prioritize, and temporize threats to life. ${ }^{1}$ Accordingly, none of these authors are arguing against its importance. However, these multidisciplinary resuscitation practitioners, educators and researchers have all experienced situations where the PS was not the appropriate first step, or the step of primary importance. Accordingly, this manuscript argues for a supplementary initial step at resuscitation time zero, which we call the 'zero point survey' (ZPS) or the pre-primary survey.

We believe it is time to reassert the importance of scene management and to challenge the primacy of the PS in hospital-based resuscitation. This is because (working in different countries and specialties) we have independently found it increasingly necessary to preemptively prepare the team, the environment and the equipment. In other words, when time permits, the first step in resuscitation is not always 'airway, breathing, and circulation. Importantly, the PS does not explicitly address non-technical factors such as self, team, environment and equipment, but fortunately the ZPS does. We have not yet provided empiric supportive evidence for the ZPS, but provocatively, nor have proponents of the traditional PS.

Just like the PS, this multidisciplinary author group has designed the ZPS such that it could be applied to each of our resuscitation environments: prehospital, emergency department, operating theater and intensive care unit. This common structure is important because the ZPS, like the PS, should facilitate communication and action both within teams and between teams. Both the PS and ZPS offer a distillation of common sense and structure to mitigate potential chaos. Both also offer the all-important first step that creates the subsequent resuscitation roadmap. Therefore, it is worth comparing the well-established PS and our putative ZPS in terms of which seems most fit for task.

On occasion, resuscitation requires nothing more than opening the airway, administering oxygen, or providing vasopressor support. In these cases, a small team can prioritize the 'airway, breathing, and circulation' with all haste. However, resuscitative form should follow resuscitative function, and we believe that the ZPS provides a cognitive roadmap better suited to modern medical reality. We are increasingly managing complex frail patients, large teams, and changing personnel. There is also a greater understanding of the importance of human factors, and the threat from diagnostic ambiguity, competing priorities, and cognitive bias. Accordingly, our shared mental models need to address both teamprocesses and task-based processes, and both technical factors and non-technical factors. The period before first patient contact is an opportunity to appraise environmental, cognitive and teambased cues. While unequivocal medical data are lacking, there is extensive literature from many high-stakes professions that preplanning increases team resilience and successful outcome.

In prehospital medicine, protocols typically mandate a focused and preemptive evaluation of scene hazards and key personnel. ${ }^{2}$ Similar benefits are possible no matter the resuscitation location, or the clinical specifics. Assessment of self, team, and environment could occur at the start of a clinical shift, immediately prior to the arrival of a critically ill patient, or be revisited periodically during the resuscitation. Regardless, the ZPS would serve as a structured evaluation. It could help maintain situation awareness by bolstering attention, perception and comprehension of the current environment. This in turn should make it easier to predict future priorities and concerns. ${ }^{3,4}$ This could facilitate problem-identification and error-avoidance, which is particularly important as situations become more complex, unfamiliar or dynamic. ${ }^{5-8}$ Furthermore, understanding and adapting to the environment may facilitate better placement of people and equipment. This in turn could optimize the order and efficiency of critical first steps in both patient assessment and stabilization.

\section{ZERO POINT SURVEY: THE SPECIFICS OF A PRE-PRIMARY SURVEY}

Summarized in Fig. 1, the ZPS is intended to interrogate and manage resuscitation prior to the PS. The ZPS provides a structured

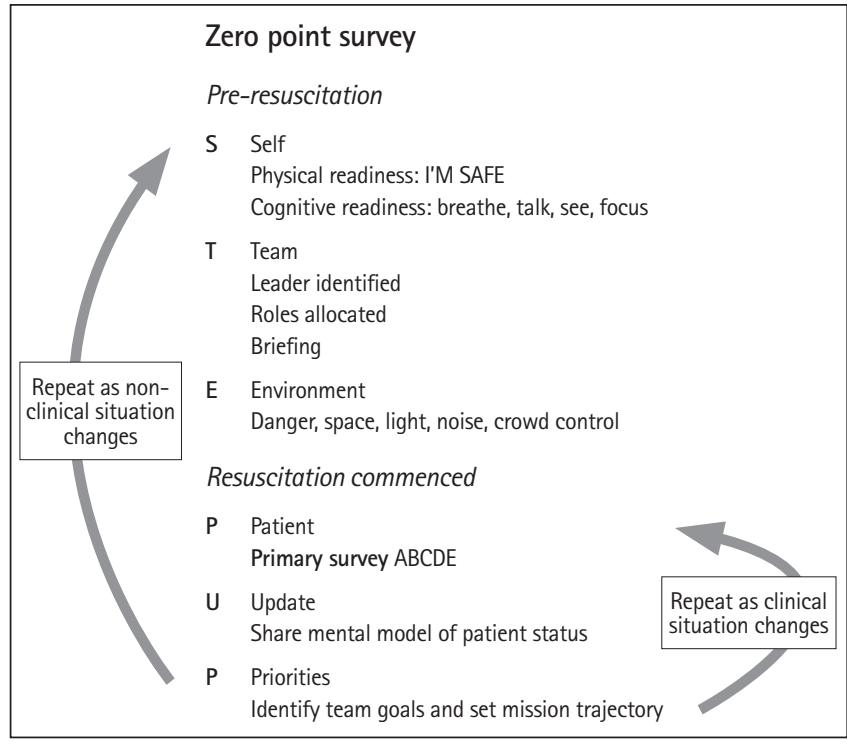

Fig. 1. How the zero point survey may optimize non-clinical processes before and during a resuscitation. I'M SAFE, illness, medication/other drugs, stress, alcohol, fatigue, eating/elimination; $A B C D E$, airway, breathing, circulation, disability, exposure. 
approach to optimizing preparedness for the individual (the self check), the resuscitation team (the team brief), and the environment (the environmental scan), prior to the hands-on PS.

\section{Self check}

Prior to hands-on interventions, team members should optimize psychological preparation, or mental readiness for engagement. This can be divided into one's own internal readiness, and that of the team.

Personal readiness requires physical and cognitive preparedness. A rapid physical readiness checklist is provided by the I'M SAFE (illness, medication/other drugs, stress, alcohol, fatigue, eating/elimination) acronym. ${ }^{9}$

While the time immediately before a resuscitation may not provide the ideal opportunity to confront one's psychological shortcomings or biases, it is possible despite even minimal warning (e.g., following an alert from the ambulance service) to empty one's bladder or ingest a snack. Such small measures to optimize personal performance speak to the concept of additive marginal gains: small steps in preparation, when summed together, which can produce large advances in performance.

Especially in high stress settings, cognitive readiness may be enhanced using simple techniques that minimize autonomic hyper-arousal, such as controlled breathing ${ }_{1}^{10,11}$ reframing threats as challenges, reminding oneself of previous training, and reviewing available resources (guidelines, checklists, equipment, and team members). ${ }^{12}$ Active visualization or mental rehearsal can help team members predict and prepare for likely next steps, which in turn helps to establish conditions in which the desired outcome is more likely to be achieved. ${ }^{13}$ Similarly, team members can imagine or verbalize a specific routine or chosen keyword to focus attention during task commencement. This combination of controlled breathing, positive self-talk, imagery, and triggering to action has been summarized as 'breathe, talk, see, focus', and has been comprehensively described in a recent emergency medicine publication that includes authors of this manuscript. ${ }^{14}$

\section{Team brief}

Ensuring team readiness includes identifying a team leader and allocating roles. Using a small amount of preparatory information (e.g., a trauma patient's age, mechanism of injury and field vital signs), mental models can be developed and shared for both taskwork (what will need to be done) and teamwork (how the team will work together to complete the tasks). ${ }^{15}$ Once the resuscitation is underway, team performance can be optimized when the team leader periodically verbalizes a mental model of patient status (update) and team goals (priorities). ${ }^{16}$

\section{Environmental scan}

A rapid environmental scan can help to identify safety threats, and provides an opportunity to optimize safety and adequacy of the workspace. We have summarized this under the headings of danger, space, light, noise and crowd in Fig. 1. Obvious dangers such as a combative patient, exposed body fluids, and slip/trip hazards should be identified and mitigated.

The ability to quickly establish and control 'resuscitative real estate' differentiates expert from novice paramedics. ${ }^{17}$ This rapid cognitive appraisal involves assessing the current situation, visualizing alternatives and judging which options are likely to benefit (versus harm) the patient or team. A similar approach can be used to survey the hospital resuscitation environment. Suitable questions include "Is there enough space to safely manage this patient?" and "If not, should we remove things away from the patient or move the patient away from these things?"

Early and frequent environmental assessments decrease the likelihood that clinical logistics will be compromised by space restrictions. Removing obstacles may also help mitigate the risk of injury to team members (tripping, collisions, and sharps injury). Similarly, the patient may benefit from being moved or repositioned (e.g., to enhance airway management and the likelihood of first-pass intubation). High performing resuscitation teams will be aware of and anticipate the need for effective lighting, reducing unnecessary noise pollution, ${ }^{18}$ and crowd control..$^{19}$ Communication is key and can include structured commands to nonparticipants, such as "behind the line."

Just as resuscitators are trained to repeat the PS when faced with change or uncertainty, we advocate for repeating the ZPS. This can be triggered whenever there are critical interventions to 'airway, breathing, or circulation', or a sudden change in environment occurs (equipment failure, arrival of a second victim). As such, the ZPS can be seen as complementary to the PS, promoting a team's ability to adapt and respond to dynamic or unpredictable events.

A structured approach to the ZPS is summarized by the 'STEP UP' acronym (Fig. 1), in which $\mathrm{P}$ (primary survey/patient assessment) is delayed until self, team, and environmental factors have been controlled. 'UP' provides both leaders and team members with a reminder to share updates $(U)$ of patient status, and the immediate goals of treatment to establish the desired clinical trajectory (P, priorities).

\section{CONCLUSION}

Non-technical skills and human factors are increasingly recognized as critical ingredients in the success or failure of acute care 
delivery in a number of high stakes clinical domains. ${ }^{20-23}$ This is reflected in the evolution of life support courses, which now incorporate components of the ZPS. For example, the European Trauma Course emphasizes team briefs and equipment checks, ${ }_{1}^{24}$ and the Advanced Pediatric Life Support Course manual contains the I'M SAFE checklist. ${ }^{25}$ However our collective experience of debriefing hundreds of real and simulated resuscitations in four nations suggests a need for a memorable and structured approach to addressing non-technical preparation.

Although our ZPS concepts are likely appreciated by experienced practitioners, they are still not widely or explicitly taught. Our shared experience is that: (1) these concepts are not well remembered or demonstrated in the resuscitations and simulations that we witness; (2) adequate control of the environment (space, light, noise, crowd) is incomprehensively taught or practiced in life support courses; (3) there is a need for in-hospital teams (including ward based rapid response teams, intensive care unit teams, staff on acute medicine wards) to share a common approach and language; (4) currently no memorable approach or acronym exists that concisely encompasses this spectrum of non-technical skills; (5) while the literature addresses many components of leadership, communication, and teamwork, it often underemphasises the importance of controlling environmental factors.

The PS is a 'tried and tested' approach that provides structure to the clinical aspects of resuscitation, and we are not suggesting it be removed. Rather, we believe there are safety and performance benefits to performing an antecedent supplementary ZPS, which can set the stage for expert team behaviors from the outset of patient contact to the completion of resuscitation. The goal of the ZPS is to increase the likelihood of patient rescue and to help create and maintain robust resuscitation teams. Regardless, good ideas are not enough, and data must follow. It behooves us as resuscitationists, educators and researchers to evaluate the effectiveness of the ZPS, the PS, and all aspects of resuscitation science. In the meantime, there is face-validity to the idea that, like many beloved films, the PS could also benefit from a prequel.

\section{CONFLICT OF INTEREST}

No potential conflict of interest relevant to this article was reported.

\section{REFERENCES}

1. Driscoll P, Skinner D. ABC of major trauma: initial assessment and management. I. Primary survey. BMJ 1990;300:1265-7.

2. Wilson $M H_{1}$ Habig $K$, Wright $C$, Hughes $A$, Davies $G$, Imray $C H$.
Pre-hospital emergency medicine. Lancet 2015;386:2526-34.

3. Endsley M. Toward a theory of situation awareness in dynamic systems. Hum Factors 1995;37:32-64.

4. Shrestha L, Prince C, Baker D, Salas E. Understanding situation awareness: concepts, methods, training. Hum Technol Interact Complex Syst 1995;7:45-83.

5. Stanton N, Chambers P, Piggott J. Situation awareness and safety. Saf Sci 2001;39:189-204.

6. Endsley M. A taxonomy of situation awareness errors. In: Fuller R, Johnson N, McDonald N, editors. Human factors in aviation operations. Avebury: Aldershot; 1995. p.287-92.

7. Hartel $C$, Smith K, Prince $C$. Defining aircrew situation awareness: searching for mishaps with meaning. In: Jensen $D$, editor. Proceedings of the 6th International Symposium on Aviation Psychology. Columbus, OH: Ohio State University; 1991.

8. Gaba DM, Howard SK, Small SD. Situation awareness in anesthesiology. Hum Factors 1995;37:20-31.

9. Wikipedia. IMSAFE [Internet]. San Francisco, CA: Wikimedia Foundation; 2016 [cited 2017 Mar 7]. Available from: https:// en.wikipedia.org/wiki/IMSAFE.

10. Mesagno C, Mullane-Grant T. A comparison of different preperformance routines as possible choking interventions. J Appl Sport Psychol 2010;22:343-60.

11. Anton NE, Montero PN, Howley LD, Brown C, Stefanidis D. What stress coping strategies are surgeons relying upon during surgery? Am J Surg 2015;210:846-51.

12. Moore $\amalg$, Vine $S J$, Wilson MR, Freeman P. Examining the antecedents of challenge and threat states: the influence of perceived required effort and support availability. Int J Psychophysiol 2014;93:267-73.

13. Lorello GR, Hicks CM, Ahmed SA, Unger Z, Chandra D, Hayter MA. Mental practice: a simple tool to enhance team-based trauma resuscitation. CJEM 2016;18:136-42.

14. Lauria MJ, Gallo IA, Rush S, Brooks J, Spiegel R, Weingart SD. Psychological skills to improve emergency care providers' performance under stress. Ann Emerg Med 2017;70:884-90.

15. Lim BC, Klein KJ. Team mental models and team performance: a field study of the effects of team mental model similarity and accuracy. J Organ Behav 2006;27:403-18.

16. Westli HK, Johnsen BH, Eid J, Rasten I, Brattebo G. Teamwork skills, shared mental models, and performance in simulated trauma teams: an independent group design. Scand J Trauma Resusc Emerg Med 2010;18:47.

17. Campeau A. Introduction to the "space-control theory of paramedic scene management". Emerg Med J 2009;26:213-6.

18. Folscher LL, Goldstein LN, Wells M, Rees D. Emergency department noise: mental activation or mental stress? Emerg Med J 
2015;32:468-73.

19. Kelleher DC, Kovler ML, Waterhouse L, Carter EA, Burd RS. Factors affecting team size and task performance in pediatric trauma resuscitation. Pediatr Emerg Care 2014;30:248-53.

20. Larsson J, Holmstrom IK. How excellent anaesthetists perform in the operating theatre: a qualitative study on non-technical skills. Br J Anaesth 2013;110:115-21.

21. Flowerdew L, Brown R, Vincent C, Woloshynowych M. Identifying nontechnical skills associated with safety in the emergency department: a scoping review of the literature. Ann Emerg Med 2012;59:386-94.

22. Haerkens MH, Kox M, Lemson J, Houterman S, van der Ho- even JG, Pickkers P. Crew Resource Management in the Intensive Care Unit: a prospective 3-year cohort study. Acta Anaesthesiol Scand 2015;59:1319-29.

23. Steinemann S, Berg B, DiTullio A, et al. Assessing teamwork in the trauma bay: introduction of a modified "NOTECHS" scale for trauma. Am J Surg 2012;203:69-75.

24. Gwinnutt C, Driscoll P, Grunfeld M, et al. European Trauma Course: the team approach. Niel: European Resuscitation Council; 2017.

25. Samuels M, Wieteska S; Advanced Life Support Group. Advanced paediatric life support: a practical approach to emergencies. 6th ed. Chichester: Wiley-Blackwell; 2016. 